\title{
Space Magnetism and Superconductivity: Diamagnetic Expulsion, Meissner Effect, Magnetic Pressure and Quantum Trapping Lead to the Origin and Stability of the Saturn Rings
}

\author{
Vladimir V. Tchernyi (Cherny) ${ }^{1}$, Andrey Yu. Pospelov², Sergey V. Kapranov³ \\ ${ }^{1}$ Modern Science Institute, SAIBR, Moscow, Russia \\ ${ }^{2}$ Independent Researcher, Los Angeles, USA \\ ${ }^{3}$ A.O. Kovalevsky Institute of Marine Biological Research, RAS, Sevastopol, Russia \\ Email: chernyv@mail.ru
}

How to cite this paper: Tchernyi (Cherny), V.V., Pospelov, A.Yu. and Kapranov, S.V. (2019) Space Magnetism and Superconductivity: Diamagnetic Expulsion, Meissner Effect, Magnetic Pressure and Quantum Trapping Lead to the Origin and Stability of the Saturn Rings. Journal of Applied Mathematics and Physics, 7, 1625-1636. https://doi.org/10.4236/jamp.2019.78110

Received: June 10, 2019

Accepted: August 9, 2019

Published: August 12, 2019

\begin{abstract}
Existence of the magnetic field of Saturn and the temperature around $70-100$ $\mathrm{K}$ nearby of it bring us to the idea of diamagnetism and superconductivity of the rings particles. The rings could emerge from the icy particles moving on chaotic orbits around Saturn within protoplanetary cloud. After appearance of the magnetic field of Saturn all chaotic orbits of icy particles start to shift to the magnetic equator plane, where there is a minimum of the particles magnetic energy, due to diamagnetic force of expulsion like Meissner phenomenon. Each particle comes to the stable position preventing its own horizontal and vertical shift. Particles are locked within three-dimensional magnetic well due to Abrikosov quantum vortex phenomenon for superconductor. This mechanism is valid and it works even particles have a small fraction of superconductor. Final picture is similar to the picture of iron particles forms the same shape around a magnet on laboratory table. Any other phenomena like gravity resonances, dusty plasma and others may bring some peculiarities to the final picture of the rings. It follows that magnetic field of Saturn and low temperature around of it are the main reason for the rings origin and the rings is product of the early time of the magnetic field of Saturn appearance. Additional matter to the rings also may come from the frozen water particles generated from the Saturn sputniks geysers due to magnetic coupling between planet and satellites. The data of Cassini mission to Saturn rings are conforming suggested theory of their origin and existence.
\end{abstract}

\section{Keywords}

Saturn Rings Origin, Space Magnetism, Space Diamagnetism, Space Superconductivity, Space Ice 


\section{Introduction}

NASA had made four missions to Saturn: Pioneer, Voyager-1 and -2 and outstanding Cassini (2004-2017). But the best answer of the rings problem is given by P. Estrada, R.H. Durisen and J.N. Cuzzi: "After the Cassini grand finale, is there a final consensus on ring origin and age?" [1]. Since G. Galileo in 1610 discovered Saturn rings there are three version of this process. Two of them devoted to gravity defragmentation when massive object was tidally disrupted when passing nearby Saturn [2] [3]. Cassini found no iron in the rings and to support gravity defragmentation theory NASA scientists decided to use Titan-sized icy satellite [3]. This theory could not be considered as entirely completed. Because it could not explain why rings exist only for the planets behind the asteroid belt; why there is a particles separation; thin, width and sharp edges of the rings; spokes in the $B$ ring; electromagnetic and other phenomena of the rings.

J. C. Maxwell, 1859 proved the rings consist of an infinite number of separated particles. Cassini found rings particles consist of $93 \%$ of ice and $7 \%$ of carbon. Our third version of the rings origin is based on Safronov theory of planets formation and evolution of the protoplanetary cloud [4]. At the beginning of the process of formation of the rings system all iced particles are rotating around Saturn in accordance with Kepler's law on chaotic orbits inside the protoplanetary cloud.

Our main thesis is the rings appeared as a result of the interaction of iced particles of the protoplanetary cloud with the magnetic field of Saturn. After appearance of the magnetic field of Saturn all particles start to interact with it. The question is how all orbits of the particles may come to the magnetic equator plane of Saturn. If we are able to find answer to this question so it means that the disk of the rings particles is a product of the early days of the Saturn magnetic field.

Low temperature near Saturn and presence of the magnetic field bring us to idea of the importance of particles diamagnetism and superconductivity. The high-temperature superconductivity was discovered by J. G. Bednorz and K. A. Muller in 1986 [5]. Diamagnetism and superconductivity of ice was demonstrated by A. N. Babushkin et al. in 1986 [6] and F. Yen, T. Gao [7]. Superconductivity of C36 has been conjectured in 1998 [8]. In 2011 B. Almog from the G. Deutscher group has demonstrated how the small size of superconductor disk frozen with liquid nitrogen and conjugated together with exceptional large scale diamagnetic material is trapped in a surrounding magnetic field and it can be made to hover over a magnet in any position with any movements [9]. So, even $7 \%$ of a glassy carbon superconductor composition of Saturn rings particles which is located within $93 \%$ of diamagnetic frozen water ice may contribute to their behavior like a type II superconductor. It is well known fact the Meissner effect is observed in type II superconductors even for sufficiently weak magnetic fields. 
An interesting idea of the rings formation is coming from the general electromagnetic theory physics when we notice the similarity of the picture of the Saturn rings to the picture when iron particles creates dense and rarefied regions in a nonuniform magnetic field near by the magnet on laboratory table (Figure 1).

To demonstrate how rings particles disk appeared due to diamagnetism and superconductivity of the iced particles we will follow our consideration presented in [10]-[25].

\section{Electromagnetic Modeling of the Rings Formation}

Origin of the Rings from the Iced Particles of the Protoplanetary Cloud. With emergence of the Saturn magnetic field the icy particles of the protoplanetary cloud begin to demonstrate diamagnetism and all of them become involved in an additional azimuth-orbital motion. Placing the origin of the coordinates at the center of the planet and directing the $\mathrm{z}$ axis along the magnetic moment of the planet (orthogonal to equator) $-\mu$, the expression for magnetic energy is:

$$
U_{H}=\frac{R^{3} \mu^{2}}{r^{6}}\left(3 \cos ^{2} \theta+1\right)
$$

Here superconducting sphere of the radius $R$ located within the protoplanetary cloud at the distance $r$, the angle $\theta$ between the vector $r$ and the axis $z$. From expression follows the magnetic energy of the superconducting particle has a minimum value when the radius-vector $r$ is in a plane of the magnetic equator and is perpendicular to the axis $z(\cos \theta=0)$. Consider now only one particle. Evidently its azimuth-orbital direction trajectory (orbit) can only be disturbed by the magnetic field. However in case of a significant amount of particles forming the protoplanetary cloud, after a transient time estimated as 1000 years or more, collisions between particles will compensate their azimuth-orbital movements, and as a result all orbits of the particles of the protoplanetary cloud

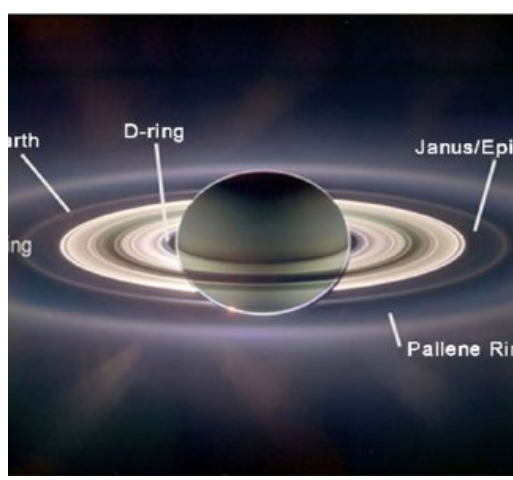

(a)

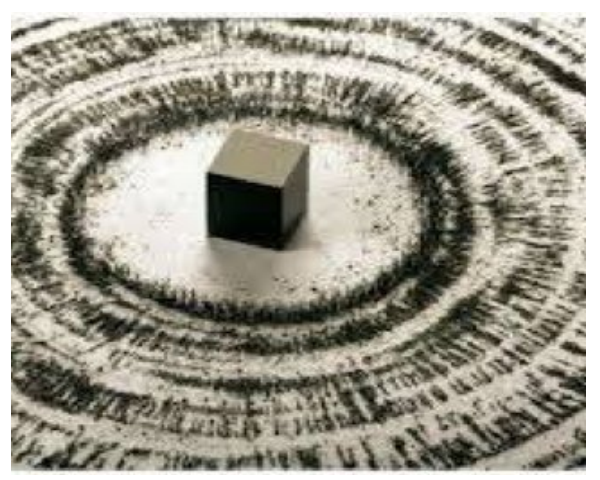

(b)

Figure 1. (a) https://saturn.jpl.nasa.gov/resources/ Image and Title Credit: NASA/JPL/ JHUAPL/University of Colorado/Central Arizona College/SSI. Saturn rings system; (b) https://wadevenden.files.wordpress.com/2014/07/magnet.jpg?w=500 Iron particles create dense and rarefied regions in nonuniform magnetic field near by the magnet on laboratory table. 
should come together to the magnetic equator plane and create highly flattening disc around the planet. Within the disc of the rings all particles will become located on the Kepler's orbit where there is a balance of gravity, centrifugal and electromagnetic forces.

Repelling and Collision of Particles inside the Width of the Rings. We can estimate an interaction of two superconducting particles for two different cases. The first one is when two particles located in the same plane within the sombrero of the rings $(z=0)$, and the second situation is when two particles are located on the different planes but on the same axis $(\rho=0)$. For the particles with the magnetic moments $\mu_{1 z}$ and $\mu_{2 z}$ located on the same plane, $z=0$, we get the interaction energy as:

$$
U=\frac{\mu_{1 z} \mu_{2 z}}{\rho^{3}}
$$

From it follows that both particles will repel each other and they will maintain a separate distance between them. This result has been confirmed by the data of Cassini mission: the particles are separated. If particles are located on the same axis but on different planes, the expression for the interaction energy is:

$$
U=-\frac{\mu_{1 z} \mu_{2 z}}{|z|^{3}}
$$

Now both particles could attract each other; they could even collide or stick together and form bigger clusters or lumps of ice. This process has an experimental conformation by the Cassini mission.

\section{Electromagnetic and Quantum Phenomena Related to Saturn Rings Origin}

Thin Width and Sharp Edges of the Rings. Diamagnetic particles collapse into the stable system of rings as a result of their exchange between the areas of the gradient of magnetic field within the plane of the magnetic equator with the force: $F=-\mu d H / d z$, where $\mu$-the magnetic moment of the particle, $d H / d z$-the gradient of the magnetic field along the $\mathrm{z}$ axis of the magnetic dipole. The force of the diamagnetic expulsion forms sharp edges of the ring: $F=-\mu d H / d y$, where $d H / d y$ - the gradient of the magnetic field along the radius of the ring. The accidental break in the ring will be stabilized by the force of the diamagnetic expulsion $F=-\mu d H / d x$, where $d H / d x$-gradient of the magnetic field in the tangential direction. The magnetic field in the plane of the rings disc is nonuniform because each superconducting particle is push out magnetic field of its own volume. Magnetic field lines will strive to go through the region with the highest magnetic permeability. It is known that the superconducting powders gather in areas with low density of the magnetic field. Density gradient flow of the magnetic field repels superconducting particles of each other, and also cleans the gaps within the rings system and forms a rigid thin structure of the separated rings. The density of the magnetic flow inside each ring will be lower than within 
surrounding its space. The difference of density of the flow will cause directed inward magnetic pressure on the each ring, therefore, the rings have sharp edges.

During interaction of the diamagnetic-superconducting iced particles of the protoplanetary cloud with magnetic field of Saturn all their chaotic orbits start to shift to magnetic equator plane of Saturn and protoplanetary cloud finally is collapsing into the rings disk (Figure 2).

Radial Dust Flow. Superconducting material is characterized by the London's penetration depth $\lambda_{\mathrm{L}}$ of the magnetic field. Smaller particles do not couple to the planetary magnetic field because they lose their superconductivity due to their small size. The dynamics of these particles is different from the dynamics of the particles with larger size, $>2 \lambda_{\mathrm{L}}$. Small particles will fall down to the planet due to gravity. Thus, existence of a radial planetary dust flux composed of submicron's size particles is related to a lack of superconductivity of the ring particles due to their small size. It is also possible for the particles to lose their superconductivity by collisions and by magnetic field fluctuations.

The Azimuth Brightness of the Saturn $A$ Ring. Present understanding of this phenomenon is based on: 1) an assumption of a synchronous rotation of the ring's particles with their asymmetrical form as extended ellipsoids directed under a small angle to the orbit; 2) existence of an asymmetrical albedo of the surface. Consider now our model for this phenomenon. If the superconductor is placed in the magnetic field, a magnetic moment directed oppositely to the external field is induced. The matter is magnetized not along the external magnetic field but in the opposite direction. A superconductive rings particle in the form of the rod attempts to align itself perpendicularly to the magnetic field lines. It is a known fact from science of ice [26] that growing snowflakes at the temperature below $-22^{\circ} \mathrm{C}$ take the form of prisms. Thus, the prism of the superconducting ice particle will be oriented perpendicularly to the field lines of the poloidal and toroidal components of the magnetic fields of the Saturn. It becomes now clear that the variable azimuth brightness of the Saturn's rings system $A$ is related to the orientation of the elongated ellipsoid of the superconducting particles versus the direction of the planetary magnetic field.

Spokes in the $\boldsymbol{B}$ Rings System. M. Hedman and P. Nicholson [27] have received understanding the matter of the $B$ ring of Saturn's demonstrates for the observer an optical illusion. $B$ ring of Saturn contain three times less matter than previously thought. "The best analogy is something like a fog over the meadow

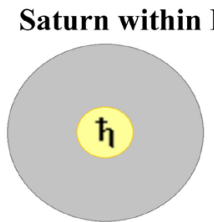

(a)

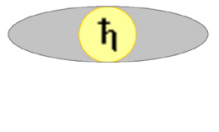

(b)

\section{Saturn with Rings Disk}

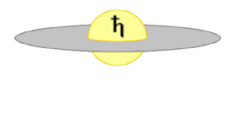

(c)

Figure 2. Transformation of protoplanetary cloud into rings disk after appearance of the magnetic field of Saturn due to interaction of iced particles with it within cloud. From (a) to $(\mathrm{c})$. 
may seem less transparent and empty, than a water-filled pool, which has a much higher density than the fog" (P. Nicholson). The spokes of the $B$ ring are aligned almost radially. The size of the spokes is about $10^{4} \mathrm{~km}$ along the radius and about $10^{3} \mathrm{~km}$ along the orbit of the rings. The spectral radiation power of spokes provides a specific periodicity about $640.6 \pm 3.5 \mathrm{~min}$ which almost coincides with the period of rotation of the magnetic field of Saturn $(639.4 \mathrm{~min})$. There is a strong correlation of maxima and minima of activity of spokes with the spectral magnetic longitudes which is connected to presence or absence of the radiation of Saturn's Kilometric Radiation. When the particles enter into anomalous region the diamagnetic expulsion force that is applied to the particles changes its value. The particles then begin to change their orbit. For the significant number of participating particles, for the external observer, this process appears as the turbulent cloud stretched along the radius in the form of spokes. After passing anomaly, particles return to their prior orbit and the common appearance of the rings is recovered.

Wide Band Pulse Radiation of the Rings. Data of Voyager have shown that the rings radiate intrinsic wide band pulse radiation within the $20 \mathrm{KHz}-40.2$ $\mathrm{MHz}$. When superconducting particles approaching distance about $10^{-8} \mathrm{~m}$ or if they have a point contact, a superconducting transition can occur, as electrons can be tunneled through the gap. Consequently, this type of superconducting weak link begins to generate electromagnetic radiation-a non-stationary Josephson phenomenon for superconductors. The radiation frequency is proportional to the junction voltage, $v=2 \mathrm{eV} / \mathrm{h}$, where $2 \mathrm{e} / \mathrm{h}=483,6 \mathrm{MHz} / \mu \mathrm{V}$, e is the charge of electron, $\mathrm{h}$ is the Plank constant.

Frequency Anomalies of Thermal Radiation of the Rings. Cassini Composite Infrared Spectrometer (CIRS) spatially resolved Saturn's main rings in the far-infrared, measuring the spectrum between $25 \mu \mathrm{m}$ and $0.5 \mathrm{~mm}$. A spectral roll-off below $200 \mu \mathrm{m}$ for each of the A, B and C rings was found (Figure 3(a)) [28]. From these data temperatures and emissivities for each ring were derived. Interpretation of Cassini CIRS spectral roll-off in Saturn's rings has encountered difficulties.

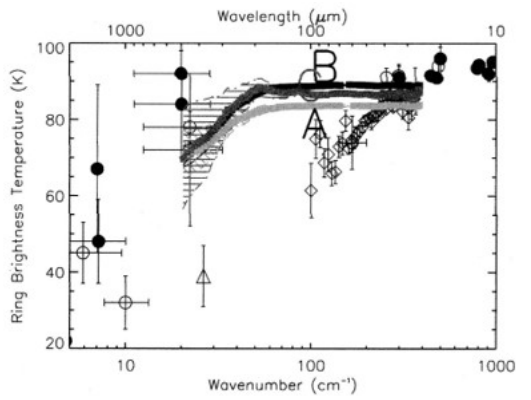

(a)

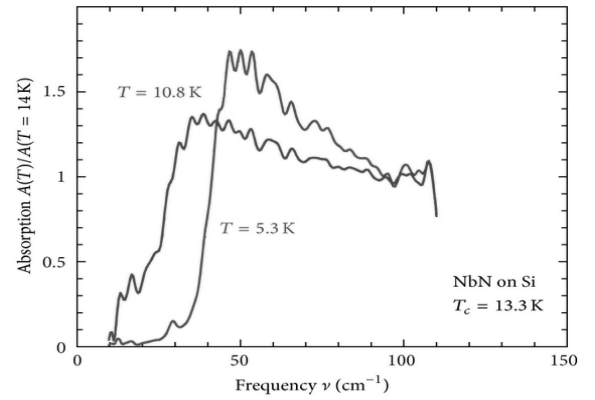

(b)

Figure 3. (a) Brightness temperatures of the A, B and C rings as a function of spectra are shown; (b) Absorption spectrum of the superconductor. M. Dressel. Electrodynamics of Metallic superconductor. Advances in Condensed Matter Physics. Vol. 2013.

https://www.hindawi.com/journals/acmp/2013/104379/. 
From the comparison of Figure 3(a) and Figure 3(b) we see the same behavior of the spectral dependence for Saturn's rings and for the superconductor. It follows the Saturn rings particles could possess superconducting matter.

Quantum Phenomenon of the Saturn Rings Particles. Recently was demonstrated an important fact that even small amount of superconducting matter may hold more than 70,000 times its own weight [9]. The experimental set up of how high temperature superconductivity is working on the best way B. Almog presented in Chicago at the ASTC Conference in 2011. It is based on theoretical discovery of A. Abrikosov of the vortex structure as a quantum nature of the superconductor [29]. In case of Saturn rings we do have similar situation to the case shown above. The rings particles contain $93 \%$ of ice and only $7 \%$ of carbon. It means that superconductivity will be responsible for the rings particles disk formation due to contain only $7 \%$ of carbon.

An interaction of type II superconductor with external magnetic field is a quantum physics phenomenon. The magnetic field inside superconductor behaves like a quantum particle, like a quantum object. And as a fact magnetic field is magnetized inside superconductor and strands of lines of the magnetic field remain inside superconductor, they get trapped inside superconductor (Figure 4). Some of the flux line becomes to be pin, and they never could take move. The reason for that is that superconductor doesn't like magnetic fields lines moving around. What it actually does, it locks them in place. By doing that it blocks itself. The action of locking prevents superconducting particles of the rings from moving within the disc. It is quantum trapping, quantum locking and quantum levitation. Also the action of trapping prevents the disc itself from moving in space.

For the Saturn disk of rings the problem of stability of the pinning structure of the superconducting iced particles is important. An exact mathematical solution of it was presented by I.M. Sigal, T. Tzaneteas in 2013 [30] and in 2015 [31] on the bases of Ginzburg-Landau equations which play a key role in superconductivity and particle physics.

Consequently superconducting iced particles will be locked within the rings disc of the magnetic equator plane by the Saturn magnetic field due to phenomena of quantum locking, quantum trapping and quantum levitation. The rings disk itself will be suppressed by the magnetic pressure from both sides along the $\mathrm{z}$ axis, because along a meridian the magnetic energy becomes to be bigger on the distance from the minimum value at the magnetic equator. Finally we have a magnetic well of the rings disc which is sufficiently disturbing picture of Saturn magnetic field lines in the area of magnetic equator, Figure 5.

As a result each particle of the rings disk of Saturn is locked strong enough within three-dimensional magnetic well. Thus it means we have a stable enough structure of the rings disk which is stabilized by the magnetic force on the vertical direction due to the gradient of magnetic flux and on the horizontal direction by the quantum trapping and quantum pinning of the Abrikosov vortex structure for each particle. 


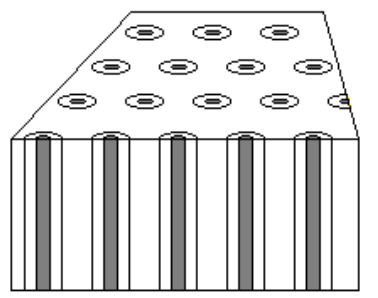

(a)

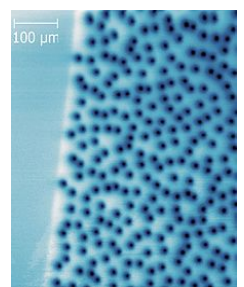

(b)

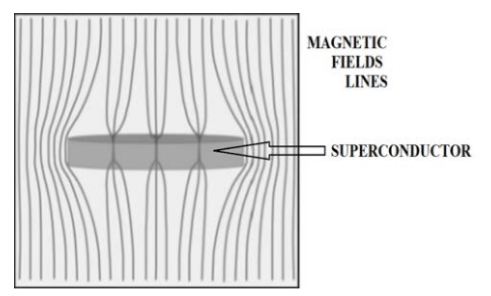

(c)

Figure 4. (a) Magnetic field is magnetized inside superconductor. Some of the flux line becomes to be pin, they don't move. This type superconductor allows only partial expulsion of a magnetic field. The volume of it is permeated with a kind of strands inside (dark lines), "Abrikosov vortex" (a fluxon), where the superconductor becomes a normal conductor. But the whole sample behaves like a superconductor (http://makeitquantum.ru/superconductor-in-magnetic-field/); (b) This is a real photo of the surface of a 200-nm-thick YBCO superconducting film with flux pinning structure taking by scanning SQUID microscopy. Huge amount of magnetic field lines penetrating sample of superconductor (vortices) and hold it against any perpendicular horizontal movements (Imaged by Wells, F. S., Pan, A. V., Wang, R., Fedoseev, S. A. and Hilgenkamp, H. (2015). Analysis of low-field isotropic vortex glass containing vortex groups in YBa2Cu3O7-x thin films visualized by scanning SQUID microscopy. Scientific Reports, 5 , 8677 (https://www.nature.com/articles/srep08677); (c) Natural superconducting iced particle of the rings which is located along the horizontal equator plane is interacting with vertical Saturn's magnetic field lines. We can see that some of magnetic lines will be expelled outside of the sample of superconductor of the second group. And at the same time some of them will remain inside the sample and superconductor locks this magnetic field flux lines inside itself (https://en.wikipedia.org/wiki/Flux_pinning).

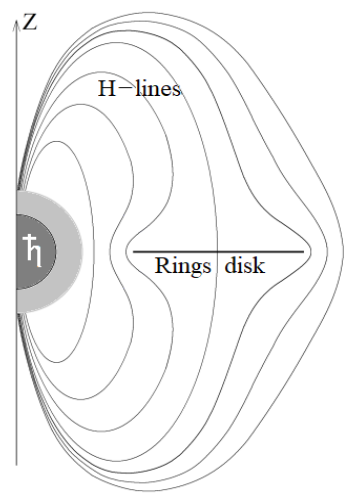

(a)

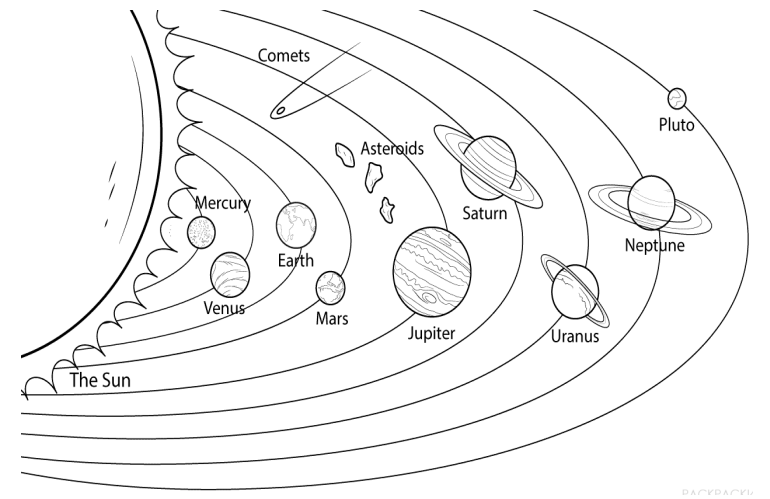

(b)

Figure 5. (a) Thus particles within the rings disc become to be locked by the quantum locking along the horizontal line; (b) Saturn axis is tilted with respect to its orbit around the Sun, which is similar to Earth's tilt. The Saturn rings are located in the magnetic equator plane. The Earth has no rings because of enough heating by the Sun.

Also there is a probability of contribution to the matter of the rings disc from the debris of the asteroids, moons and satellites migrated inward towards Saturn and coming superconducting meteorites. Another situation may happen when particles of the frozen water coming from the geyser of the geologically active satellite go to the rings disc due to magnetic coupling between Saturn and its satellite (Figure 6). 


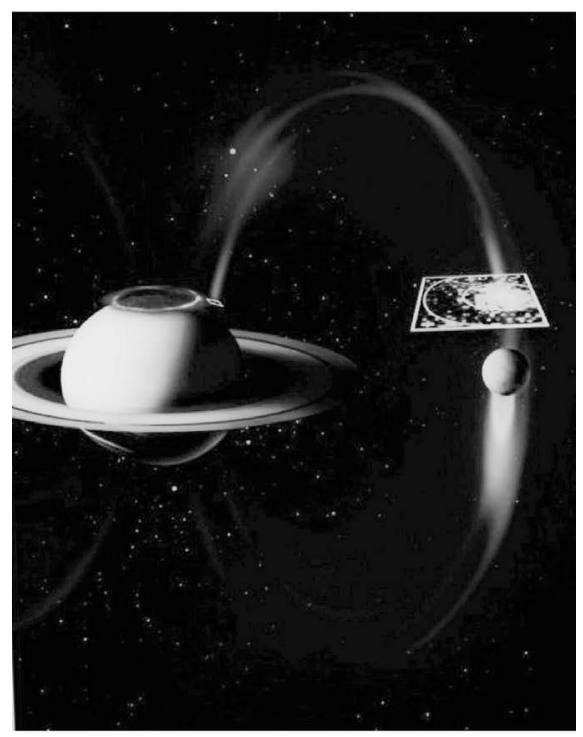

Figure 6. https://saturn.jpl.nasa.gov/resources/5289/PIA13765. April 20, 2011. Image and Title Credit: NASA/JPL/JHUAPL/University of Colorado/Central Arizona College/SSI. Electrical circuits between Saturn and Enceladus. The magnetic field also provides a path for electrons to flow as illustrated in the graphic above. There is a magnetic coupling between Saturn and Enceladus.

An important discovery was made in 2005. The Cassini spacecraft started multiple close flybys of Enceladus and discovered water-rich plumes venting from the south polar region [32]. Cryovolcanoes near the south pole shoot geyser-like jets of water vapor up to $500 \mathrm{~km}$, molecular hydrogen, other volatiles, and solid material, including sodium chloride crystals and ice particles, into space, totaling about $200 \mathrm{~kg}$ per second [33]. Over 100 geysers have been identified [34]. Some of the water vapor falls back as "snow"; the rest escapes, and supplies most of the material making up Saturn's E ring [35] [36] (Figure 6). I may hint at an interaction between Saturn's magnetosphere and the torrent of particles issuing from Enceladus.

\section{Conclusions}

Surprisingly, assuming the presence of magnetic properties of diamagnetism and superconductivity in the ice particles of Saturn's rings, we can easily explain the origin of Saturn's rings, their stable existence, as well as such problems as:

- Origin, evolution, dynamics and stability of the rings.

- Why rings of Saturn located within magnetic equator plane.

- Considerable flattening and the sharp edges of the ring system.

- Why particles of the rings are separated and could collide.

- Change of the azimuth brightness of the Saturn $A$ ring particles.

- Formation of spokes in the ring $B$.

- High radio-wave reflectivity and low brightness of the rings.

- Anomalous reflection of circularly polarized microwaves.

- Spectral anomalies of thermal radiation of the rings. 
- Why planetary rings in the solar system appear only after asteroid belt.

- Why the Earth has no rings, etc.

We have demonstrated the magnetic field of Saturn, diamagnetism and superconductivity of the iced particles of the protoplanetary cloud are the most important reason of the Saturn rings origin and it existence. Also it follows that it is necessary to study matter of the space ice, natural diamagnetism and superconductivity of the space matter behind the asteroid belt.

\section{Conflicts of Interest}

The authors declare no conflicts of interest regarding the publication of this paper.

\section{References}

[1] Estrada, P.R., Durisen, R.H. and Cuzzi, J.N. (2017) Ballistic Transport: After the Cassini Grand Finale, Is There a Final Consensus on Ring Origin and Age? American Geophysical Union Meeting, New Orleans, 12 December 2017, Paper 298112.

[2] Fridman, A.M. and Gor'kavyi, N.N. (1999) Physics of Planetary Rings. Springer-Verlag, New York. https://doi.org/10.1007/978-3-662-03918-2

[3] Canup, R.M. (2010) Origin of Saturn's Rings and Inner Moons by Mass Removal from a Lost Titan-Sized Satellite. Nature, 468, 943-946.

https://doi.org/10.1038/nature09661

[4] Safronov, V.S. (1972) Evolution of the Protoplanetary Cloud and Formation of the Earth and the Planets. Nauka Press, Moscow. NASA Technical Translation F-677.

[5] Bednorz, J.G. and Müller, K.A. (1986) Possible High Tc Superconductivity in the Ba-La-Cu-O System. Zeitschrift für Physik B, 64, 189-193.

https://doi.org/10.1007/BF01303701

[6] Babushkina, G.V., Kobelev, L.Ya., Yakovlev, E.N. and Babushkin, A.N. (1986) Superconductivity of Ice Under High Pressure. Physics of Solid State, 28, 3732-3734. (In Russian)

[7] Yen, F. and Gao, T. (2015) Dielectric Anomaly in Ice near 20K; Evidence of Macroscopic Quantum Phenomena. The Journal of Physical Chemistry Letters, 6, 2822-2825. https://doi.org/10.1021/acs.jpclett.5b00797

[8] Côté, M., Grossman, J.C., Cohen, M.L. and Louie, S.G. (1998) Electron-Phonon Interactions in Solid C36. Physical Revew Letters, 81, 697-700. https://doi.org/10.1103/PhysRevLett.81.697

[9] Deutscher, G., Azoulay, M., Almog, B. and Deutscher, B. (2011) Quantum Levitation. Quantum Locking, Quantum Trapping. ASTC Conf., Maryland, 15-18 Oct. 2011. https://www.youtube.com/watch?v=4HHJv8lPERQ

[10] Pospelov, A.Yu., Tchernyi, V.V. and Girich, S.V. (1998) Planet's Rings: Super-Diamagnetic Model and New Course of Investigations. Proc. SPIE 42nd Annual meet., San Diego, CA, July 27-Aug. 1 1997, Small Spacecraft, Space Environments and Instrumentation Technologies, SPIE, 3116, 117-128. https://doi.org/10.1117/12.293330

[11] Girich, S.V., Pospelov, A.Yu. and Tchernyi, V.V. (1998) Radar Data Explanation via Superdiamagnetic Model of the Saturn's Rings. Annual Report of AAS, 30 th Meeting Division of Planetary Science, Madison, WI, 11-16 Oct. 1998, Bulletin of the American Astronomical Society, 30, 1043. 
[12] Tchernyi, V.V., et al. (1999) Are Saturn Rings Superconducting? University of Alabama, Huntsville, NASA Marshall Space Flight Center, Huntsville Space Physics Colloquium, 20 Aug.1999.

[13] Tchernyi, V.V. and Pospelov, A.Yu. (2005) Possible Electromagnetic Nature of the Saturn's Rings: Superconductivity and Magnetic Levitation. Progress in Electromagnetic Research, PIER, 52, 277-299. https://doi.org/10.2528/PIER04082801

[14] Tchernyi, V.V. and Pospelov, A.Yu. (2007) About Hypothesis of the Superconducting Origin of the Saturn's Rings. Astrophysics and Space Science, 307, 347-356. https://doi.org/10.1007/s10509-006-9054-7

[15] Tchernyi, V.V. and Chensky, E.V. (2005) Electromagnetic Background for Possible Magnetic Levitation of the Superconducting Rings of Saturn. Journal of Electromagnetic Waves and Applications, 19, 1997-2006.

https://doi.org/10.1163/156939305775570440

[16] Tchernyi, V.V. and Chensky, E.V. (2005) Movements of the Protoplanetary Superconducting Particles in the Magnetic Field of Saturn Lead to the Origin of Rings. Geoscience and Remote Sensing Letters-IEEE, 2, 445-446. https://doi.org/10.1109/LGRS.2005.852767

[17] Tchernyi, V.V. (2002) Possible Superconductivity of Saturn Rings. University of Hawaii, Institute for Astronomy, Colloquia, Spring/Summer, 7 Aug. 2002.

[18] Tchernyi (Cherny), V.V. (2009) Origin of the Saturn Rings: Electromagnetic Model of the Sombrero Rings Formation. In: Denis, J.H. and Aldridge, P.D., Eds., Space Exploration Research, Nova Science Publishers, NY, Chapter 11, 261-275.

[19] Tchernyi, V.V. (2009) To Discovery of Initial Formation (Origin) of the Sombrero Rings of Saturn: Role of Electromagnetism. International Astronomical Union, Assembly, XXVII General Assembly, 3-14 Aug. 2009, Rio de Janeiro, Brazil, Abstract Book, Symposium No. 263-Icy Bodies in the Solar System, Report No. 263, 56, 63.

[20] Tchernyi (Cherny), V.V. (2013) Could Superconductivity Contribute to the Saturn Rings Origin? Journal of Modern Physics (Special Issue on Superconducting Physics), 4, 17-23. https://doi.org/10.4236/jmp.2013.46A005

[21] Tchernyi (Cherny), V.V. (2013) About Role of Electromagnetism to the Saturn Rings Origin-To the Unified Theory of the Planetary Rings Origin. International Journal of Astronomy and Astrophysics, 3, 412-420. https://doi.org/10.4236/ijaa.2013.34049

[22] Tchernyi, V.V. and Pospelov, A.Yu. (2018) Superconductivity of Saturn Rings: Quantum Locking, Rings Disk Thickness and Its time Creation. Journal of Modern Physics, 9, 219-432. https://doi.org/10.4236/jmp.2018.93029

[23] Tchernyi, V.V. and Pospelov, A.Yu. (2018) Quantum Locking and the Meissner Effect Lead to the Origin and Stability of the Saturn Rings System. International Journal of Astronomy and Astrophysics, 8, 104-120. https://doi.org/10.4236/ijaa.2018.81008

[24] Tchernyi (Cherny), V.V., Kapranov, S.V. and Pospelov, A.Yu. (2018) Diamagnetic Expulsion as a Possible Cause of the Origin and Stability of the Saturn Rings. Physics and Astronomy International Journal, 2, 121-126. https://doi.org/10.15406/paij.2018.02.00073

[25] Pospelov, A.Yu. and Tchernyi, V.V. (2019) Energy Gap in Saturn's Rings. Journal of Modern Physics, 10, 477-485. https://doi.org/10.4236/jmp.2019.104033

[26] Maeno, N. (1981) The Science of Ice. Hokkaido University Press, Sapporo.

[27] Hedman, M.M. and Nicholson, P.D. (2016) The B-Ring's Surface Mass Density 
from Hidden Density Waves: Less than Meets the Eye? Icarus, 279, 109-124. https://doi.org/10.1016/j.icarus.2016.01.007

[28] Spilker, L.J., Pilorz, S.H., Edgington, S.G., Wallis, B.D., Brooks, S.M., Pearl, J.S. and Flazar, F.M. (2005) Earth, Moon and Planets, 96, 149-163.

https://doi.org/10.1007/s11038-005-9060-8

[29] Abrikosov, A.A. (957) On the Magnetic Properties of Superconductors of the Second Group. Soviet Physics JETF, 5, 1174-1182.

[30] Sigal, I.M. and Tzaneteas, T. (2013) On Stability of Abrikosov Lattices. 25 Aug. 2013. https://doi.org/10.1088/0951-7715/25/4/1187

[31] Sigal, I.M. and Tzaneteas, T. (2016) On Stability of Abrikosov Vortex Lattices. 12 Aug. 2016.

[32] Hansen, C.J., Esposito, L., Stewart, A.I.F., Colwell, J., Hendrix, A., Pryor, W., Shemansky, D. and West, R. (2006) Enceladus' Water Vapor Plume. Science, 311, 1422-1425. https://doi.org/10.1126/science.1121254

[33] Spencer, J.R. and Nimmo, F. (2013) Enceladus: An Active Ice World in the Saturn System. Annual Review of Earth and Planetary Sciences, 41, 693-717. https://doi.org/10.1146/annurev-earth-050212-124025

[34] Dyches, P., Brown, D., et al. (2014) Cassini Spacecraft Reveals 101 Geysers and More on Icy Saturn Moon. NASA, July 28, 2014.

[35] NASA/JPL/Space Science Institute (2015) Jump up to: Icy Tendrils Reaching into Saturn Ring Traced to Their Source. NASA News, April 14, 2015.

[36] NASA/JPL/Space Science Institute (2014) Jump up to: Ghostly Fingers of Enceladus. NASA News, Sept. 19, 2006. 